\title{
Prática insulinoterápica realizada por pessoas com diabetes na Atenção Primária em Saúde
}

\author{
Insulin therapy practice performed by people with diabetes in Primary Healthcare \\ Práctica de la terapia con insulina realizada por personas con diabetes en Atención Primaria de Salud
}

Como citar este artigo:

Cunha GH, Fontenele MSM, Siqueira LR, Lima MAC, Gomes MEC, Ramalho AKL. Insulin therapy practice performed by people with diabetes in Primary Healthcare. Rev Esc Enferm USP. 2020;54:e03620. doi: https://doi.org/10.1590/S1980-220X2019002903620

(D) Gilmara Holanda da Cunha ${ }^{1}$
(D) Marina Soares Monteiro Fontenele ${ }^{1}$
(D) Larissa Rodrigues Siqueira ${ }^{1}$
(D) Maria Amanda Correia Lima ${ }^{1}$
(D) Maria Elisa Curado Gomes ${ }^{1}$
(D) Ane Kelly Lima Ramalho ${ }^{1}$
${ }^{1}$ Universidade Federal do Ceará,
Faculdade de Farmácia, Odontologia
e Enfermagem, Departamento de
Enfermagem, Fortaleza, CE, Brasil.

e Enfermagem, Departamento de

\begin{abstract}
Objective: To analyze insulin therapy performed by people with diabetes in Primary Healthcare. Method: A cross-sectional, descriptive and quantitative study. Data collection was carried out through an interview using a form with sociodemographic, clinical and insulin therapy variables. Absolute and relative frequencies as well as prevalence ratio were calculated and the chi-squared test was used, with $\mathrm{p}<0.05$ being significant. Results: The sample consisted of 150 patients. Most were female (66.7\%), aged 50-85 years (79.3\%) and some were illiterate (16.7\%). Type 2 diabetes (62.0\%) with complications (42.7\%), and using oral hypoglycemic agents and insulin stood out. Syringes/needles (83.1\%), lancets $(85.5 \%)$, reagent strips $(91.0 \%)$ and insulin vials $(93.8 \%)$ were stored incorrectly by the majority. The correct form predominated in preparation, application and transport. Waste was disposed of incorrectly. In the general analysis most performed the insulin therapy stages inappropriately $(93.3 \%)$. Sociodemographic and clinical variables did not influence insulin therapy, but there was a significant difference in the intra-group analysis for incorrect performance in some groups. Conclusion: Insulin therapy was inappropriately performed in most cases.
\end{abstract}

DESCRIPTORS

Diabetes Mellitus; Insulin; Primary Care Nursing; Primary Health Care; Health Education. 


\section{INTRODUÇÃO}

O diabetes mellitus (DM) é uma condição crônica caracterizada por distúrbio metabólico, em que ocorre hiperglicemia persistente por defeitos na secreção ou ação da insulina, sendo o tipo 1 (DM1) e o tipo 2 (DM2) os mais comuns. O DM1 é uma doença autoimune, com destruição das células beta pancreáticas, ocasionando deficiência na produção de insulina. Já no DM2 a ação da insulina está dificultada e ocorre resistência insulínica, tendo como fatores de risco importantes o histórico familiar de DM, idade avançada, obesidade, sedentarismo, pré-diabetes ou DM gestacional ${ }^{(1)}$.

Em nível epidemiológico mundial, o DM tem representatividade pelo número crescente de acometidos e redução da qualidade de vida, estimando-se que entre 2010 e 2030 ocorra um aumento de 69,0\% no número de casos. Prospecta-se que em 2025 haverá 350 milhões de pessoas com diabetes no mundo, e que para igual período no Brasil, existam 18,5 milhões ${ }^{(2)}$.

O tratamento para DM visa o controle glicêmico, redução de complicações e melhora da qualidade de vida dos pacientes. Pessoas com DM1 precisam repor a insulina para atingirem valores basais do hormônio fisiológico. $\mathrm{O}$ tratamento do DM2 envolve mudanças no estilo de vida quanto à alimentação e exercício físico, fármacos hipoglicemiantes orais, e insulina para níveis glicêmicos não controlados por período prolongado ou descompensação metabólica ${ }^{(3)}$.

A insulinoterapia pode ser realizada com diferentes tipos de insulina (ultrarrápida, rápida, intermediária, prolongada, pré-misturas), e dispositivos com distintas características e indicações (seringa/agulha, caneta, bomba de insulina), envolvendo etapas e cuidados a serem seguidos, tais como o armazenamento, transporte, preparo, aplicação e descarte de resíduos $^{(3-5)}$. O manejo da insulinoterapia com base em práticas seguras é importante para a assistência em saúde de qualidade, devendo-se orientar usuários e cuidadores para o tratamento seguro e eficaz ${ }^{(5)}$. Porém, há barreiras para adesão à insulina pelos pacientes, incluindo o desconforto na aplicação, punções digitais diárias, além do manejo adequado de suas etapas ${ }^{(6-7)}$.

Nesse contexto, da forma em que está organizado o sistema de saúde público do Brasil, a atenção primária é responsável por acompanhar pessoas com DM, visando reduzir complicações, incapacidades e internações decorrentes da doença $a^{(7)}$. Este acompanhamento é essencial, pois a insulina é um hormônio que se manuseado inadequadamente, resulta em situações de risco e falha terapêutica ${ }^{(8)}$. Danos podem ocorrer da fase de preparo até o descarte de resíduos, devendo-se seguir orientações específicas para evitar $\operatorname{erros}^{(5)}$.

A maioria das pesquisas analisam a adesão das pessoas com DM à insulina ${ }^{(9-11)}$, mas também é relevante estudar o manejo recomendado com base na prática segura das etapas da insulinoterapia. Assim, o objetivo deste estudo foi analisar as etapas da prática insulinoterápica realizada por pessoas com DM acompanhados na atenção primária em saúde.

\section{MÉTODO}

\section{TIPO DE ESTUDO}

Estudo transversal, descritivo e quantitativo.

\section{População}

Pessoas com DM em insulinoterapia atendidas em Unidade de Atenção Primária em Saúde (UAPS) de Fortaleza, Ceará, Brasil, a qual faz parte da rede de atenção primária do Sistema Único de Saúde (SUS).

Os critérios de inclusão foram: pessoas com diagnóstico de DM1 ou DM2, acompanhadas na unidade de saúde e em insulinoterapia por pelo menos seis meses. Foram excluídas as gestantes. Para seleção de participantes foi utilizada a técnica de amostragem por conveniência, na qual as pessoas com DM em uso de insulina foram convidadas a participar da pesquisa quando compareciam à UAPS para consulta médica.

Para atender aos objetivos do estudo, a amostra foi dimensionada para estimar a prevalência de pessoas com DM em insulinoterapia, com 95\% de confiança de que o erro da estimação não ultrapassasse $5 \%$. Considerou-se que tal prevalência é desconhecida na população, sendo estipulada em 50\% (prevalência presumida), pois proporciona maior tamanho de amostra, e que havia 245 pacientes em insulinoterapia acompanhados no serviço. Para tanto, aplicou-se a seguinte expressão:

$$
n=\frac{z^{2} \cdot p \cdot(1-p) \cdot N}{\varepsilon^{2} \cdot(N-1)+z^{2} \cdot p \cdot(1-p)}
$$

Nesta fórmula, o z é igual ao valor da estatística $z(1,96)$ para o grau de confiança adotado (95\%) e p, $\mathrm{Ne} \varepsilon$ correspondem à prevalência presumida $(0,50)$, à população $(245)$ e ao erro tolerável $(0,05)$, respectivamente. Assim, foi calculada uma amostra de 150 pacientes.

\section{Coleta de Dados}

A coleta de dados ocorreu de janeiro de 2016 a dezembro de 2017, sendo realizada de forma individual, por meio de entrevista, com duração de aproximadamente 50 minutos em ambiente privativo. Utilizou-se formulário produzido a partir das diretrizes para insulinoterapia ${ }^{(5,12)}$, o qual foi submetido à pré-teste antes do início do estudo, com 30 pacientes que não compuseram a amostra, para efeito de validação do instrumento. $O$ formulário possuía duas partes: 1. Variáveis sociodemográficas e clínicas (idade, sexo, cor da pele autorreferida, escolaridade, estado civil, situação da pessoa com DM na família, situação ocupacional, número de pessoas no domicílio, renda mensal familiar, tipo de DM, tempo de diagnóstico, locais de acompanhamento em saúde, complicações relacionadas ao DM, uso de hipoglicemiantes orais e outros medicamentos, hábito tabagista, uso de álcool); 2. Etapas da insulinoterapia: 2.1 Armazenamento (local de acondicionamento de canetas, seringas/agulhas, lancetas, fitas reagentes e frasco de insulina); 2.2 Preparo (lavar as mãos, rolar o frasco de insulina para homogeneizá-la antes de aspirar, aspirar a insulina regular antes da NPH - Neutral Protamine Hagedorn); 2.3 Aplicação (limpar local de aplicação com álcool, pinçar a pele e aplicar insulina com agulha em ângulo de $90^{\circ}$, esperar 5 segundos após aplicação, mudar sistematicamente o local de aplicação); 2.4 Transporte (bagagem de mão, mala, isopor/bolsa térmica, 
exposição à luz solar/calor excessivo); 2.5 Descarte de seringas/agulhas, lancetas, fitas reagentes, frasco de insulina, algodão e canetas (garrafa polietileno tereftalato $\mathrm{PET}$, recipiente rígido resistente, recipiente próprio para perfurocortante, lixo comum).

\section{ANÁlise E TRATAMENTO DOS DADOS}

As respostas dos pacientes quanto às etapas da insulinoterapia foram analisadas em conformidade com as diretrizes vigentes ${ }^{(5,12)}$. Para o preparo e aplicação, os participantes responderam sim ou não para cada procedimento descrito. Para o armazenamento, transporte e descarte, cada resposta aos itens foi categorizada como correta ou incorreta. Considerou-se correto: armazenamento (prateleira da geladeira para canetas novas e frascos de insulina em uso, e temperatura ambiente para canetas em utilização, seringas, fitas reagentes e lancetas); transporte (bagagem de mão ou bolsa térmica/ isopor); descarte (recipiente próprio para material perfurocortante ou recipiente rígido resistente à perfuração com abertura larga e tampa, para seringas/agulhas, canetas, frascos de insulina, lancetas, fitas reagentes e algodão). Ao final, houve a classificação geral da prática insulinoterápica para cada paciente, sendo considerada adequada somente para quem realizou todas as etapas do processo corretamente.

$\mathrm{Na}$ análise estatística utilizou-se o Software Statistical Package for Social Sciences (SPSS) versão 19.0. Foram determinadas as frequências absoluta e relativa, e para verificar associação entre variáveis foi aplicado o teste qui-quadrado para as análises intragrupo e intergrupo. Estabeleceu-se um nível de significância de 5\%, considerando-se estatisticamente significante um valor de $\mathrm{p}<$ 0,05. A força de tal associação foi avaliada pela determinação da razão de prevalência e seu respectivo intervalo de confiança de $95 \%$.

\section{AspeCtos ÉTICOS}

Projeto aprovado pelo Comitê de Ética em Pesquisa da Universidade Federal do Ceará, em 14/08/2014, sob o Parecer no 751.330. Foram observadas as diretrizes da Resolução no 466/2012, do Conselho Nacional de Saúde, sobre pesquisas com seres humanos. Todos os participantes assinaram o Termo de Consentimento Livre e Esclarecido. As diretrizes para Estudo Observacional em Epidemiologia (STROBE) foram seguidas.

\section{RESULTADOS}

Dentre os pacientes, a maioria era do sexo feminino $(66,7 \%)$, faixa etária de 50 a 85 anos $(79,3 \%)$, cor da pele autorreferida parda $(62,0 \%)$, casados ou em união estável $(52,7 \%)$, renda familiar mensal de um a três salários mínimos $(60,0 \%)$, aposentados $(50,7 \%)$, com mais de três pessoas no domicílio (44,7\%), com menos de oito anos de estudo $(36,7 \%)$, ocorrendo número relevante de analfabetos $(16,7 \%)$. Dados expressos na Tabela 1.
Tabela 1 - Caracterização sociodemográfica das pessoas com diabetes mellitus - Fortaleza, CE, Brasil, 2017.

\begin{tabular}{|c|c|c|}
\hline Variáveis sociodemográficas & $\mathbf{N}$ & $\%$ \\
\hline \multicolumn{3}{|l|}{ Sexo } \\
\hline Masculino & 50 & 33,3 \\
\hline Feminino & 100 & 66,7 \\
\hline \multicolumn{3}{|l|}{ Faixa etária (em anos) } \\
\hline$\leq 29$ & 11 & 7,3 \\
\hline $30-39$ & 06 & 4,0 \\
\hline $40-49$ & 14 & 9,3 \\
\hline$\geq 50$ & 119 & 79,3 \\
\hline \multicolumn{3}{|l|}{ Cor da pele autoinformada } \\
\hline Branca & 45 & 30,0 \\
\hline Negra & 11 & 7,3 \\
\hline Parda & 93 & 62,0 \\
\hline Indígena & 01 & 0,7 \\
\hline \multicolumn{3}{|l|}{ Estado civil } \\
\hline Solteiro & 33 & 22,0 \\
\hline Casado/vive junto/união consensual & 79 & 52,7 \\
\hline Viúvo & 18 & 12,0 \\
\hline Divorciado/separado & 20 & 13,3 \\
\hline \multicolumn{3}{|l|}{ Renda mensal da família } \\
\hline$<1$ salário* & 32 & 21,3 \\
\hline 1 - 3 salários & 90 & 60,0 \\
\hline > 3 salários & 28 & 18,7 \\
\hline \multicolumn{3}{|l|}{ Situação ocupacional } \\
\hline Empregado & 30 & 20,0 \\
\hline Desempregado & 20 & 13,3 \\
\hline Aposentado & 76 & 50,7 \\
\hline Afastado & 01 & 0,7 \\
\hline Do lar & 23 & 15,3 \\
\hline \multicolumn{3}{|c|}{ Número de pessoas que moram no domicílio } \\
\hline Reside sozinho & 09 & 6,0 \\
\hline 2 & 39 & 26,0 \\
\hline 3 & 35 & 23,3 \\
\hline$>3$ & 67 & 44,7 \\
\hline \multicolumn{3}{|l|}{ Escolaridade (em anos) } \\
\hline Analfabeto & 25 & 16,7 \\
\hline$<8$ anos & 55 & 36,7 \\
\hline $8-12$ & 52 & 34,7 \\
\hline$>12$ & 18 & 12,0 \\
\hline
\end{tabular}

* Salário mínimo vigente no Brasil no período de estudo - 2016: $\mathrm{R} \$$ 880,00; 2017: R\$ 937,00.

Nota: $\mathrm{N}=150$

Maior parte da amostra era de pessoas com DM2 (62,0\%), 63 possuíam de 10-19 anos de diagnóstico e alguma complicação da doença (42,7\%), sobretudo retinopatia. A maioria $(75,3 \%)$ usava hipoglicemiantes orais, insulina e medicamentos para comorbidades $(82,0 \%)$, como losartana, sinvastatina e ácido acetilsalicílico. Deixaram de fumar após DM um total de $49(32,7 \%)$ pacientes, e 25 (16,7\%) ingeriam bebida alcoólica três vezes na semana (Tabela 2 ). 
Tabela 2 - Caracterização clínica das pessoas com diabetes mellitus - Fortaleza, CE, Brasil, 2017.

\begin{tabular}{|c|c|c|}
\hline Variáveis clínicas & $\mathbf{N}$ & $\%$ \\
\hline \multicolumn{3}{|l|}{ Tipo de DM* } \\
\hline $\mathrm{DM}^{* 1}$ & 24 & 16,0 \\
\hline $\mathrm{DM}^{*} 2$ & 93 & 62,0 \\
\hline Não soube informar & 33 & 22,0 \\
\hline \multicolumn{3}{|c|}{ Tempo de diagnóstico de $\mathrm{DM}^{*}$ (em anos) } \\
\hline $0-9$ & 54 & 36,0 \\
\hline $10-19$ & 63 & 42,0 \\
\hline $20-29$ & 22 & 14,7 \\
\hline Mais de 29 anos & 11 & 7,3 \\
\hline \multicolumn{3}{|c|}{ Complicações relacionadas ao $\mathrm{DM}^{*}$} \\
\hline Não possui complicações & 86 & 57,3 \\
\hline Cegueira/retinopatia & 19 & 12,7 \\
\hline Cardiovascular & 11 & 7,3 \\
\hline Pé diabético & 08 & 5,3 \\
\hline Nefropatia & 02 & 1,3 \\
\hline Mais de uma complicação & 24 & 16,0 \\
\hline \multicolumn{3}{|c|}{ Faz uso de agente(s) hipoglicemiantes(s) oral(is) } \\
\hline Sim & 113 & 75,3 \\
\hline Não & 37 & 24,7 \\
\hline \multicolumn{3}{|c|}{ Faz uso de outros medicamentos } \\
\hline Sim & 123 & 82,0 \\
\hline Não & 27 & 18,0 \\
\hline \multicolumn{3}{|l|}{ Hábito tabagista } \\
\hline Nunca fumou & 94 & 62,7 \\
\hline Sim & 07 & 4,7 \\
\hline Abandonou & 49 & 32,7 \\
\hline \multicolumn{3}{|l|}{ Uso de álcool } \\
\hline Nunca ingeriu & 96 & 64,0 \\
\hline Sim & 25 & 16,7 \\
\hline Não & 29 & 19,3 \\
\hline
\end{tabular}

* Diabetes mellitus.

Nota: $(\mathrm{N}=150)$.

A Tabela 3 mostra o armazenamento de insumos, preparo e aplicação da insulina. Os usuários de canetas fizeram armazenamento correto. Dos 150 pacientes, 113 usavam seringas acopladas à agulha, mas $94(83,1 \%)$ guardavam este material na geladeira para reutilização, uma prática não mais recomendada, pois seringas e agulhas devem ser desprezadas após uso. Quanto ao armazenamento de lancetas e fitas reagentes, respectivamente, $85,5 \%$ e $91,0 \%$ dos pacientes acondicionavam de modo incorreto na geladeira. Frascos de insulina em uso foram armazenados incorretamente na porta da geladeira por $93,8 \%$ dos participantes. Antes de preparar a insulina, a maioria realizava lavagem de mãos $(95,3 \%)$. Homogeneizar o frasco de insulina antes da utilização $(79,6 \%)$ e aspirar primeiro insulina regular quando em combinação com NPH $(82,9 \%)$ foi um procedimento realizado por maior parte dos indivíduos. $\mathrm{Na}$ aplicação da insulina, 86 (57,3\%) não limpavam o local com álcool, e 66,0\% da amostra esperava cinco segundos para retirar a agulha. Nos itens em que a amostra não foi 150 , os pacientes utilizavam canetas ou não associavam insulinas regular e NPH (Tabela 3).
Tabela 3 - Armazenamento da insulina e insumos, preparo e aplicação realizado por pessoas com diabetes mellitus na atenção primária em saúde - Fortaleza, CE, Brasil, 2017.

\begin{tabular}{|c|c|c|}
\hline Etapas da insulinoterapia & $\mathrm{N}$ & $\%$ \\
\hline \multicolumn{3}{|c|}{ Armazenamento de insulina e insumos } \\
\hline \multicolumn{3}{|c|}{ Canetas não utilizadas $(\mathrm{N}=37$ ) } \\
\hline Correto & 37 & 100,0 \\
\hline Incorreto & 0 & 0,0 \\
\hline \multicolumn{3}{|c|}{ Canetas sendo utilizadas $(\mathrm{N}=37)$} \\
\hline Correto & 37 & 100,0 \\
\hline Incorreto & 0 & 0,0 \\
\hline \multicolumn{3}{|c|}{ Seringas e agulhas usadas $(\mathrm{N}=113)$} \\
\hline Correto & 19 & 16,9 \\
\hline Incorreto & 94 & 83,1 \\
\hline \multicolumn{3}{|c|}{ Lancetas utilizadas no teste de glicemia $(\mathrm{N}=145)$} \\
\hline Correto & 21 & 14,5 \\
\hline Incorreto & 124 & 85,5 \\
\hline \multicolumn{3}{|c|}{ Fitas de reagentes usadas no teste glicemia $(\mathrm{N}=145)$} \\
\hline Correto & 13 & 9,0 \\
\hline Incorreto & 132 & 91,0 \\
\hline \multicolumn{3}{|c|}{ Frasco de insulina utilizado/aberto $(\mathrm{N}=113)$} \\
\hline Correto & 07 & 6,2 \\
\hline Incorreto & 106 & 93,8 \\
\hline \multicolumn{3}{|c|}{ Preparo antes da aplicação da insulina } \\
\hline \multicolumn{3}{|c|}{ Lava as mãos com água e sabão ( $N=150)$} \\
\hline $\operatorname{Sim}$ & 143 & 95,3 \\
\hline Não & 07 & 4,7 \\
\hline \multicolumn{3}{|c|}{ Rolar o frasco de insulina antes de aspirar $(\mathrm{N}=113)$} \\
\hline $\operatorname{Sim}$ & 90 & 79,6 \\
\hline Não & 23 & 20,4 \\
\hline \multicolumn{3}{|c|}{ Aspirar primeiro a regular, se associado com a insulina $\mathrm{NPH}^{*}(\mathrm{~N}=82)$} \\
\hline $\operatorname{Sim}$ & 68 & 82,9 \\
\hline Não & 14 & 17,1 \\
\hline \multicolumn{3}{|l|}{ Aplicação da insulina } \\
\hline \multicolumn{3}{|c|}{ Limpar o local de aplicação com álcool (N=150) } \\
\hline Sim & 64 & 42,6 \\
\hline Não & 86 & 57,3 \\
\hline \multicolumn{3}{|c|}{ Pinçar o local de aplicação em ângulo de $90^{\circ}(\mathrm{N}=150)$} \\
\hline $\operatorname{Sim}$ & 141 & 94,0 \\
\hline Não & 09 & 6,0 \\
\hline \multicolumn{3}{|c|}{ Esperar 5 segundos após a aplicação $(\mathrm{N}=150)$} \\
\hline Sim & 99 & 66,0 \\
\hline Não & 51 & 34,0 \\
\hline \multicolumn{3}{|c|}{ Mudar, sistematicamente, o local de aplicação $(\mathrm{N}=150)$} \\
\hline Sim & 139 & 92,7 \\
\hline Não & 11 & 7,3 \\
\hline
\end{tabular}

* NPH: Neutral Protamine Hagedorn.

Nota: $(\mathrm{N}=150)$.

A maioria $(72,6 \%)$ realizava transporte adequado das insulinas em bagagem de mão ou isopor/bolsa térmica. Quanto ao descarte dos resíduos, destacou-se que seringas e agulhas (82,3\%), lancetas $(85,5 \%)$, fitas reagentes $(91,0 \%)$, frascos de insulina $(93,8 \%)$ e canetas $(83,8 \%)$ foram descartadas incorretamente em lixo comum ou garrafa PET. Nos itens em que a amostra não foi 150 , os pacientes não utilizavam o insumo referido, conforme a Tabela 4. 
Tabela 4 - Transporte de insulina e descarte dos resíduos pelas pessoas com diabetes mellitus atendidas na atenção primária em saúde - Fortaleza, CE, Brasil, 2017.

\begin{tabular}{|c|c|c|}
\hline Etapas da insulinoterapia & $\mathbf{N}$ & $\%$ \\
\hline \multicolumn{3}{|l|}{ Transporte da insulina } \\
\hline Correto & 109 & 72,6 \\
\hline Incorreto & 41 & 27,4 \\
\hline \multicolumn{3}{|c|}{ Descarte dos resíduos da insulinoterapia } \\
\hline \multicolumn{3}{|l|}{ Seringas e agulhas ( $N=113)$} \\
\hline Correto & 20 & 17,7 \\
\hline Incorreto & 93 & 82,3 \\
\hline \multicolumn{3}{|l|}{ Lancetas $(\mathrm{N}=145)$} \\
\hline Correto & 21 & 14,5 \\
\hline Incorreto & 124 & 85,5 \\
\hline \multicolumn{3}{|l|}{ Fitas reagentes $(\mathrm{N}=145)$} \\
\hline Correto & 13 & 9,0 \\
\hline Incorreto & 132 & 91,0 \\
\hline \multicolumn{3}{|l|}{ Frascos de insulina $(\mathrm{N}=113)$} \\
\hline Correto & 07 & 6,2 \\
\hline Incorreto & 106 & 93,8 \\
\hline \multicolumn{3}{|l|}{ Algodão (N=146) } \\
\hline Correto & 137 & 93,8 \\
\hline Incorreto & 09 & 6,2 \\
\hline \multicolumn{3}{|l|}{ Canetas $(\mathrm{N}=37)$} \\
\hline Correto & 06 & 16,2 \\
\hline Incorreto & 31 & 83,8 \\
\hline
\end{tabular}

Nota: $(\mathrm{N}=150)$.

$\mathrm{Na}$ análise conjunta das etapas da insulinoterapia de cada paciente, constatou-se que a maioria realizava o processo de forma inadequada (140; 93,3\%). Apenas 10 pessoas com DM $(6,7 \%)$ realizavam todas as etapas corretamente. $\mathrm{Na}$ análise intergrupo não houve significância estatística na associação entre variáveis sociodemográficas e clínicas com o manejo adequado ou inadequado da insulinoterapia. $\mathrm{Na}$ análise intragrupo, houve diferença estatisticamente significante para participantes com idade igual ou maior a 50 anos, em que a maioria realizava insulinoterapia de forma inadequada $(p<0,0001)$. Para pacientes do sexo feminino, casados, em situação ocupacional sem rendimento, renda familiar igual ou maior a um salário mínimo, utilizando mais de três medicamentos e sem comorbidades, a maioria realizava insulinoterapia inadequadamente $(p<0,0001)$, de acordo com a Tabela 5 .

Tabela 5 - Associação das variáveis sociodemográficas e clínicas com as etapas da insulinoterapia - Fortaleza, CE, Brasil, 2017.

\begin{tabular}{|c|c|c|c|c|c|c|c|}
\hline \multirow{3}{*}{ Variáveis } & \multicolumn{4}{|c|}{ Insulinoterapia } & \multirow{3}{*}{$\begin{array}{l}\text { Valor de } p^{*} \\
\text { intragrupo }\end{array}$} & \multirow{3}{*}{$\mathrm{RP}^{\ddagger}(\mathrm{IC} \S 95 \%)$} & \multirow{3}{*}{$\begin{array}{l}\text { Valor de } p^{+} \\
\text {intergrupo }\end{array}$} \\
\hline & \multicolumn{2}{|c|}{ Adequado } & \multicolumn{2}{|c|}{ Inadequado } & & & \\
\hline & $\mathbf{N}$ & $\%$ & $\mathrm{~N}$ & $\%$ & & & \\
\hline Idade (em anos) & & & & & & & 0,102 \\
\hline$<50$ & 0 & 0 & 30 & 100,0 & - & & \\
\hline$\geq 50$ & 10 & 8,3 & 110 & 91,7 & $<0,0001$ & & \\
\hline Sexo & & & & & & & 0,817 \\
\hline Masculino & 3 & 6 & 47 & 94 & 0,206 & $0,857(0,231-3,174)$ & \\
\hline Feminino & 7 & 7 & 93 & 93 & $<0,0001$ & $1,011(0,925-1,1104)$ & \\
\hline Estado civil & & & & & & & 0,001 \\
\hline Solteiro & 0 & 0 & 33 & 100,0 & - & & \\
\hline Casado/união estável & 2 & 2,5 & 77 & 97,5 & $<0,0001$ & $0,12(0,027-0,485)$ & \\
\hline Divorciado/viúvo & 8 & 21,1 & 30 & 78,9 & $<0,0001$ & $1,235(1,044-1,460)$ & \\
\hline Escolaridade (em anos) & & & & & & & 0,662 \\
\hline$<8$ & 6 & 7,5 & 74 & 92,5 & 0,527 & $1,313(0,386-4,463)$ & \\
\hline$\geq 8$ & 4 & 5,7 & 66 & 94,3 & 0,499 & $0,981(0,901-1,068)$ & \\
\hline Situação ocupacional & & & & & & & 0,502 \\
\hline Com rendimento & 8 & 7,5 & 98 & 92,5 & 0,058 & $1,66(0,367-7,509)$ & \\
\hline Sem rendimento & 2 & 4,5 & 42 & 95,5 & $<0,0001$ & $0,969(0,890-1,054)$ & \\
\hline
\end{tabular}




\begin{tabular}{|c|c|c|c|c|c|c|c|}
\hline \multirow{3}{*}{ Variáveis } & \multicolumn{4}{|c|}{ Insulinoterapia } & \multirow{3}{*}{$\begin{array}{l}\text { Valor de } p^{*} \\
\text { intragrupo }\end{array}$} & \multirow{3}{*}{$\operatorname{RP}^{\ddagger}\left(I C^{\S} 95 \%\right)$} & \multirow{3}{*}{$\begin{array}{l}\text { Valor de } p^{+} \\
\text {intergrupo }\end{array}$} \\
\hline & \multicolumn{2}{|c|}{ Adequado } & \multicolumn{2}{|c|}{ Inadequado } & & & \\
\hline & $\mathbf{N}$ & $\%$ & $N$ & $\%$ & & & \\
\hline Renda familiar mensal & & & & & & & 0,915 \\
\hline$<1$ salário-mínimo & 2 & 6,3 & 30 & 93,8 & 0,058 & $0,922(0,206-4,129)$ & \\
\hline$\geq 1$ salários-mínimos & 8 & 6,8 & 110 & 93,2 & $<0,0001$ & $1,006(0,908-1,113)$ & \\
\hline Diagnóstico DM"| (anos) & & & & & & & 0,076 \\
\hline$<10$ anos & 1 & 1,9 & 53 & 98,1 & 0,011 & $0,198(0,026-1,517)$ & \\
\hline$\geq 10$ anos & 9 & 9,4 & 87 & 90,6 & 0,004 & $1,083(1,006-1,116)$ & \\
\hline Complicações DM"I & & & & & & & 0,402 \\
\hline $\operatorname{Sim}$ & 3 & 4,7 & 61 & 95,3 & 0,206 & $0,576(0,155-2,141)$ & \\
\hline Não & 7 & 8,1 & 79 & 91,9 & 0,128 & $1,038(0,955-1,128)$ & \\
\hline Número de medicações & & & & & & & 0,189 \\
\hline$<3$ & 1 & 2,4 & 41 & 97,6 & 0,011 & $0,286(0,037-2,186)$ & \\
\hline$>3$ & 9 & 8,3 & 99 & 91,7 & $<0,0001$ & $1,065(0,989-1,147)$ & \\
\hline Outras comorbidades & & & & & & & 0,495 \\
\hline $\operatorname{Sim}$ & 9 & 7,3 & 114 & 92,7 & 0,011 & $1,976(0,261-14,946)$ & \\
\hline Não & 1 & 3,7 & 26 & 96,3 & $<0,0001$ & $0,962(0,880-1,052)$ & \\
\hline
\end{tabular}

*Análise intragrupo realizada pelo Teste qui-quadrado com valor de $p_{;}{ }^{\dagger}$ Análise intergrupo realizada pelo Teste qui-quadrado com valor de $p ;{ }^{\ddagger}$ RP: razão de prevalência; ${ }^{\S} I C$ : intervalo de confiança; "DM: diabetes mellitus

\section{DISCUSSÃO}

Ao se analisar a prática insulinoterápica das pessoas com DM, constatou-se que a maioria realizava as etapas inadequadamente. Quanto a isso, estudos afirmam que as características sociodemográficas e clínicas influenciam nesse processo ${ }^{(5,13-17)}$. Houve prevalência de pacientes do sexo feminino, corroborando com a atual conjuntura que mostra o aumento de DM em mulheres, sobretudo, por estas procurarem mais o serviço de saúde que os homens, acrescendo as chances de diagnóstico ${ }^{(13)}$. A maioria dos participantes tinha mais de 50 anos, porque o DM2 é mais comum em maiores de 40 anos, devido ao excesso de peso, sedentarismo e histórico familiar de $\mathrm{DM}^{(14)}$. Destacaram-se pessoas de cor parda, mas não há pesquisas que relacionem a cor da pele ou etnia com DM, sobretudo, pela miscigenação existente no Brasil(15). Quanto à rede social de apoio, a maioria não residia sozinha, estando casados ou em união estável. Assim, ter familiares ou rede social é um suporte que facilita o seguimento da terapêutica ${ }^{(16)}$. Porém, estudo mostrou que se a pessoa com DM tiver muitas atribuições em casa, como cuidar de crianças/idosos, tendem a não realizar adequadamente a insulinoterapia por falta de tempo ${ }^{(13)}$.

Aposentados e com renda mensal de um a três salários mínimos prevaleceram no estudo. Pesquisa mostra que ser aposentado facilita a insulinoterapia, pois os que trabalham podem ter dificuldades relacionadas aos horários e manuseio da insulina fora da residência ${ }^{(17)}$. Estudos ainda mostram que pessoas com DM de baixa renda têm menor controle da doença e mais comorbidades ${ }^{(18-20)}$. Em relação à escolaridade, os resultados foram semelhantes aos de um estudo realizado em Portugal, em que maior parte dos pacientes tinha menos de oito anos de estudo, havendo também analfabetos, afirmando que ter maior escolaridade facilita o tratamento ${ }^{(21)}$.

O tempo de diagnóstico de DM mais referido foi de 10 a 19 anos. Este é um fator importante no acompanhamento dos pacientes, devido à associação entre duração da doença e terapêutica, com desenvolvimento de complicações micro e macrovasculares do $\mathrm{DM}^{(22)}$, ressaltando-se que a maioria possuía ao menos uma complicação do DM, configurando um importante agravo de saúde. Frequentemente, o DM associa-se a outras condições, como hipertensão arterial sistêmica e dislipidemias, corroborando com os achados desta pesquisa, em que a maioria utilizava além dos hipoglicemiantes orais e insulina, fármacos para comorbidades ${ }^{(23)}$. Alguns participantes eram tabagistas e consumiam bebida alcoólica, o que interfere negativamente na insulinoterapia, pois aumenta o risco de complicações do DM, sendo também fator de risco para outras doenças cardiovasculares ${ }^{(23)}$.

$\mathrm{Na}$ análise das etapas da insulinoterapia, o armazenamento de seringas, fitas reagentes, lancetas e frascos de insulina foram realizados de forma incorreta, sobretudo as seringas acopladas à agulha, com o intuito de reutilização. Esta prática não é mais recomendada, devido às lesões cutâneas que podem causar lipodistrofia, o que pode interferir na inoculação e ação adequada do hormônio, haja vista a imprevisibilidade da absorção da insulina em locais com lipohipertrofia ${ }^{(24)}$. A reutilização de seringas e agulhas pode ocorrer pela falta de insumos, e apesar de registros de maior disponibilidade desses materiais nas UAPS, estes podem não ser suficientes para atender às demandas da população ${ }^{(7)}$. Frascos de insulina abertos foram armazenados erroneamente pela maioria, na porta da geladeira, podendo interferir negativamente na biodisponibilidade da insulina, alterando o controle glicêmico adequado ${ }^{(5)}$.

Nas etapas de preparo e aplicação da insulina, a maioria realizou os procedimentos corretamente, mas durante o preparo, alguns não realizavam a homogeneização do frasco de insulina e não aspiravam primeiro a insulina regular em caso de combinação com a NPH, podendo reduzir a efetividade do hormônio e causar respostas clínicas indesejáveis ${ }^{(25)}$. 
Quanto à limpeza do local de aplicação com álcool, um estudo mostrou que a desinfecção geralmente não é necessária quando as aplicações são realizadas em ambientes não institucionais, como residências ${ }^{(24)}$. Ainda nesta etapa, a maioria dos participantes realizava rodízio no local de aplicação, o que reduz complicações como lipodistrofia e descontrole glicêmico ${ }^{(26)}$. Os pacientes realizavam a prega cutânea para aplicação com agulha em ângulo de $90^{\circ}$, para evitar a injeção da insulina no tecido muscular, mas quase metade das pessoas com DM não esperavam os cinco segundos para retirar a agulha do local, podendo haver refluxo de insulina, com redução da dose e do efeito esperado ${ }^{(24)}$.

Neste estudo, nenhum participante expôs o hormônio à luz solar, havendo transporte correto, evidenciando a prática atrelada à literatura ${ }^{(5,24)}$. Na última etapa, que envolve o descarte de insumos, parcela significativa desprezou canetas, seringas/agulhas, lancetas, fitas reagentes e frascos de insulina incorretamente, usando lixo comum ou garrafa PET. $\mathrm{O}$ descarte adequado seria em coletor para perfurocortantes $\left(\right.$ Descartex $\left.^{\oplus}\right)$, ou recipientes como frascos de amaciante de roupas, com tampa, boca larga e material resistente à perfuração ${ }^{(17)}$.

Em estudo realizado em São Paulo, os enfermeiros foram os principais responsáveis por orientar sobre o descarte, contudo, as orientações eram insuficientes, sendo necessária a adoção de um protocolo específico para descarte de resíduos em domicílio ${ }^{(27)}$. O descarte inadequado representa um risco para os familiares dos pacientes, sobretudo, quando há crianças no domicílio, além da população e meio ambiente, pois estes materiais podem propagar Vírus da Imunodeficiência Humana (HIV), da Hepatite B e C, necessitando-se de orientação para empoderar os pacientes no processo terapêutico ${ }^{(28)}$. Ademais, familiares podem ser orientados para auxiliar no descarte.

A realização da insulinoterapia foi inadequada para a maioria das pessoas com DM, porque estas não realizavam corretamente todas as etapas para a devida eficácia terapêutica. Diante disso, apesar de este não ser um estudo intervencionista, evidencia-se a necessidade de práticas de educação em saúde para o autocuidado, pois os pacientes podem receber informações pontuais numa consulta e não conseguirem incorporar tudo o que foi orientado pelos profissionais de saúde ${ }^{(29)}$. A estratégia de grupo de apoio pode dar continuidade ao ensino-aprendizagem, pois aumenta os acertos no processo insulinoterápico, contribuindo para $o$ autocuidado. Ademais, tem-se a estratégia de ensino por telefone, que evidenciou melhora na competência da pessoa com DM para aplicação de insulina ${ }^{(25)}$.

Neste estudo, as variáveis sociodemográficas e clínicas não influenciaram na insulinoterapia, entretanto nos grupos com idade igual ou maior a 50 anos, sexo feminino, casados, em situação ocupacional sem rendimento, com renda familiar igual ou maior a um salário mínimo, utilizando acima de três medicamentos e sem comorbidades, houve significativa diferença na realização da insulinoterapia, que foi feita de forma mais inadequada. Apesar disso, em outras pesquisas a maior escolaridade e número de pessoas no domicílio foram associados com uma prática insulinoterápica mais correta ${ }^{(16,21)}$. Em contrapartida, alguns pacientes apesar do maior nível de instrução, não conseguem compreender e seguir as medidas de tratamento. Uma justificativa é que compreender e seguir orientações de saúde é algo complexo, que está além do nível de escolaridade, transcorrendo pelo letramento funcional em saúde de cada indivíduo ${ }^{(30)}$. Tal fato pode relacionar-se também às dificuldades no relacionamento terapêutico, haja vista que o profissional passa a ser um educador em saúde, e a resolução de vieses dependem da compreensão dos questionamentos individuais dos pacientes, devendo-se adaptar a cada realidade em particular ${ }^{(29)}$.

Tem-se como limitação a validade externa do estudo, a qual pode ser restringida devido à participação de indivíduos acompanhados em uma única instituição de saúde. No entanto, esta pesquisa contribui para a assistência em saúde das pessoas com DM, pois aponta a necessidade de atividades educativas no contexto da prática insulinoterápica, voltadas tanto para os pacientes, como para seus familiares e cuidadores. Destaca-se ainda, a necessidade de pesquisas que possam avaliar a efetividade dessas estratégias educativas.

\section{CONCLUSÃO}

A maioria das pessoas com DM acompanhadas na atenção primária em saúde realizava a prática insulinoterápica inadequadamente, o que pode interferir na eficácia e segurança do processo. As variáveis sociodemográficas e clínicas não influenciaram na adequabilidade da insulinoterapia, mas constatou-se que os maiores de 50 anos, mulheres, casados, com renda familiar igual ou superior a um salário mínimo, utilizando acima de três medicamentos e sem comorbidades realizavam a insulinoterapia de forma mais inadequada.

Atividades de educação em saúde para pessoas com DM devem ser implementadas na atenção primária, com o intuito de melhorar o autocuidado, pois ao empoderar o indivíduo acerca do processo saúde-doença, contribui-se para o manejo adequado da insulinoterapia no domicílio. Destaca-se a importância de os profissionais de saúde criarem grupos de apoio aos pacientes, assim como realizar visita domiciliar, que é fundamental para acompanhamento, sobretudo, daqueles indivíduos com limitação física ou cognitiva. Os familiares e cuidadores também devem estar inseridos para auxiliar no gerenciamento da insulinoterapia.

Ademais, a equipe multiprofissional de saúde, que engloba enfermeiros, médicos e farmacêuticos, deve estar capacitada para identificar as dúvidas ou necessidades dos pacientes, a fim de orientar adequadamente as etapas da insulinoterapia dentro de seu âmbito de atuação na Unidade de Atenção Primária à Saúde. 
clínicas e etapas da insulinoterapia. Foram calculadas frequências absoluta e relativa, razão de prevalência e foi usado o teste de quiquadrado, sendo significante o p < 0,05. Resultados: A amostra foi composta de 150 pacientes. A maioria era do sexo feminino (66,7\%), faixa etária de 50-85 anos (79,3\%) e havia analfabetos (16,7\%). Destacou-se o diabetes tipo 2 (62,0\%) com complicações (42,7\%), em uso de hipoglicemiantes orais e insulina. Seringas/agulhas $(83,1 \%)$, lancetas $(85,5 \%)$, fitas reagentes $(91,0 \%)$ e frascos de insulina $(93,8 \%)$ foram armazenados incorretamente pela maioria. No preparo, aplicação e transporte predominou a forma correta. Resíduos foram descartados incorretamente. Na análise geral das etapas da insulinoterapia, a maioria a realizava de forma inadequada (93,3\%). Variáveis sociodemográficas e clínicas não influenciaram na prática insulinoterápica, mas na análise intragrupo houve diferença significante para realização incorreta em alguns grupos. Conclusão: A insulinoterapia foi realizada de forma inadequada na maioria dos casos.

\section{DESCRITORES}

Diabetes Mellitus; Insulina; Enfermagem de Atenção Primária; Atenção Primária à Saúde; Educação em Saúde.

\section{RESUMEN}

Objetivo: Analizar la insulinoterapia realizada por personas con diabetes en la Atención Primaria en Salud.Método: Estudio transversal, descriptivo y cuantitativo. La recolección de los datos fue realizada por medio de la entrevista, utilizando formulario con variables sociodemográficas, clínicas y etapas de la insulinoterapia. Fueran calculadas frecuencias absoluta y relativa, razón de prevalencia y fue utilizado la prueba de qui-cuadrado, siendo significante el p < 0,05. Resultados: La amuestra fue composta de 150 pacientes. La mayoría era del sexo femenino (66,7\%), rango de edad de 50-85 años (79,3\%) y hubo analfabetos (16,7\%). Destacó se la diabetes tipo 2 (62,0\%) con complicaciones $(42,7 \%)$, en el uso de hipoglicemiantes orales y insulina. Seringas/agujas $(83,1 \%)$, lancetas $(85,5 \%)$, tiras reactivas $(91,0 \%)$ y frascos de insulina $(93,8 \%)$ fueran almacenados incorrectamente por la mayoría. En el preparo, aplicación y transporte predominó la forma correcta. Residuos fueran descartados incorrectamente. En el análisis general de las etapas de la insulinoterapia, la mayoría realizaba de forma inadecuada (93,3\%). Variables sociodemográficas y clínicas non influenciaran en la práctica de la terapia de insulina, pero en el análisis intragrupo hubo diferencia significante para realización incorrecta en algunos grupos. Conclusión: La insulinoterapia fue realizada de forma inadecuada en la mayoría de los casos.

\section{DESCRIPTORES}

Diabetes Mellitus; Insulina; Enfermería de Atención Primaria; Atención Primaria de Salud; Educación en Salud.

\section{REFERÊNCIAS}

1. Skyler JS, Bakris GL, Bonifacio E, Darsow T, Eckel RH, Groop L, et al. Differentiation of diabetes by pathophysiology, natural history, and prognosis. Diabetes. 2017;66(2):241-55. DOI: https://doi.org/10.2337/db16-0806

2. Lima LR, Funghetto SS, Volpe CRG, Santos WS, Funez MI, Stival MM. Quality of life and time since diagnosis of diabetes mellitus among the elderly. Rev Bras Geriatr Gerontol. 2018;21(2):180-90. DOI: http://dx.doi.org/10.1590/1981-22562018021.170187

3. Thrasher J. Pharmacologic management of type 2 diabetes mellitus: available therapies. Am J Med. 2017;130( Suppl 6):S4-17. DOI: https://doi.org/10.1016/j.amjmed.2017.04.004

4. Frid AH, Kreugel G, Grassi G, Halimi S, Hicks D, Hirsch LJ et al. New insulin delivery recommendations. Mayo Clin Proc. 2016;91(9):123155. DOI: https://doi.org/10.1016/j.mayocp.2016.06.010

5. Sociedade Brasileira de Diabetes. Diretrizes da Sociedade Brasileira de Diabetes: 2017-2018. São Paulo: A.C. Farmacêutica; 2018.

6. Bhandari P, Kim M. Self-care behaviors of nepalese adults with type 2 diabetes: a mixed methods analysis. Nurs Res. 2016;65(3):202-14. DOI: 10.1097/NNR.0000000000000153

7. Neves RG, Duro SMS, Muniz J, Castro TRP, Facchini LA, Tomasi E. Estrutura das Unidades Básicas de Saúde para atenção às pessoas com diabetes: ciclos I e II do Programa Nacional de Melhoria do Acesso e da Qualidade. Cad Saúde Pública. 2018;34(4):e00072317. DOI: http://dx.doi.org/10.1590/0102-311x00072317

8. Instituto para Práticas Seguras no Uso de Medicamentos. Medicamentos potencialmente perigosos. Boletim ISMP. 2015;4(3):1-5.

9. Yavuz DG, Ozcan S, Deyneli O. Adherence to insulin treatment in insulin-naïve type 2 diabetic patients initiated on different insulin regimens. Patient Prefer Adherence. 2015;9:1225-31. DOI: https://doi.org/10.2147/PPA.S87935

10. Gerada Y, Mengistu Z, Demessie A, Fantahun A, Gebrekirstos K. Adherence to insulin self administration and associated factors among diabetes mellitus patients at Tikur Anbessa specialized hospital. J Diabetes Metab Disord. 2017;16:28. DOI: https://doi.org/10.1186/s40200-017-0309-3

11. Bermeo-Cabrera J, Almeda-Valdes P, Riofrios-Palacios J, Aguilar-Salinas CA, Mehta R. Insulin adherence in type 2 diabetes in Mexico: behaviors and barriers. J Diabetes Res. 2018;2018:3190849. DOI: https://doi.org/10.1155/2018/3190849

12. Sociedade Brasileira de Diabetes. Diretrizes da Sociedade Brasileira de Diabetes: 2015-2016. São Paulo: A.C. Farmacêutica; 2016.

13. Gusmai LF, Novato TS, Nogueira LS. The influence of quality of life in treatment adherence of diabetic patients: a systematic review. Rev Esc Enferm USP. 2015;49(5):839-46. DOI: http://dx.doi.org/10.1590/S0080-623420150000500019

14. Iser BPM, Malta DC, Ducan BB, Moura L, Vigo A, Schmidt MI. Prevalence, correlates, and description of self-reported diabetes in Brazilian capitals - results from a telephone survey. PLoS One. 2014;9(9):e108044. DOI: https://doi.org/10.1371/journal.pone.0108044

15. Campos LA. Socialismo Moreno, Conservadorismo Pálido? Cor e recrutamento partidário em São Paulo e Rio de Janeiro nas Eleições de 2012. Dados. 2015;58(3):689-719. DOI: http://dx.doi.org/10.1590/00115258201556

16. Silva EFF, Ferreira CMM, Pinho L. Risk factors and complications in type 2 diabetes outpatients. Rev Assoc Med Bras. 2017;63(7):621-7. DOI: http://dx.doi.org/10.1590/1806-9282.63.07.621

17. Cunha GH, Barbosa RVA, Fontenele MSM, Lima MAC, Franco KB, Fechine FV. Insulin therapy waste produced in the households of people with diabetes monitored in primary care. Rev Bras Enferm. 2017;70(3):646-53. DOI: http://dx.doi.org/10.1590/0034-7167-2016-0406

18. Lamy S, Ducros D, Dimeglio C, Colineaux H, Fatin R, Berger E, et al. Disentangling the influence of living place and socioeconomic position on health services use among diabetes patients: a population based study. PLoS One. 2017;12(11):e0188295. DOI: https://doi.org/10.1371/journal.pone.0188295 
19. Fosse-Edorh S, Fagot-Campagna A, Detournay B, Bihan H, Eschwege E, Gautier A, et al. Impact of socio-economic position on health and quality of care in adults with type 2 diabetes in France: the Entred 2007 study. Diabete Med. 2015;32(11):1438-44. DOI: https://doi.org/10.1111/dme.12783

20. Sortsø C, Lauridsen J, Emneus M, Green A, Jensen PB. Social inequality in diabetes patients'morbidity patterns from diagnosis to death - a Danish register based investigation. Scand J Public Health. 2018;46(1):92-101. DOI: https://doi.org/10.1177/1403494817713648

21. Caetano IRCS, Santiago LM, Marques M. Impact of written information on control and adherence in type 2 diabetes. Rev Assoc Med Bras. 2018;64(2):140-7. DOI: http://dx.doi.org/10.1590/1806-9282.64.02.140

22. Cortez DN, Reis IA, Souza DAS, Macedo MML, Torres HC. Complications and the time of diagnosis of diabetes mellitus in primary care. Acta Paul Enferm. 2015;28(3):250-5. DOI: http://dx.doi.org/10.1590/1982-0194201500042

23. Flor LS, Campos MR, Oliveira AF, Schramm JMA. Diabetes burden in Brazil: fraction attributable to overweight, obesity, and excess weight. Rev Saúde Pública. 2015;49:29. DOI: https://doi.org/10.1590/S0034-8910.2015049005571

24. Frid AH, Kreugel G, Grassi G, Halimi S, Hicks D, Hirsch LJ, et al. New insulin delivery recommendations. Mayo Clin Proc. 2016;91(9):1231-55. DOI: https://doi.org/10.1016/j.mayocp.2016.06.010

25. Pereira FGF, Diógenes MAR, Ataíde MBC, Mendonça Júnior JO, Leal DE, Xavier ATF. Fatores relacionados à utilização de insulina em diabéticos acompanhados pela Estratégia Saúde da Família. Rev APS. 2016;19(1):58-66.

26. Gentile S, Strollo F, Ceriello A. Lipodystrophy in insulin-treated subjects and other injection-site skin reactions: are we sure everything in clear? Diabetes Ther. 2016;7(3):401-9. DOI: https://doi.org/10.1007/s13300-016-0187-6

27. Andre SCS, Takayanagui AMM. Orientações sobre o descarte de resíduos gerados em domicílios de usuários de insulina. Rev Baiana Saúde Pública. 2015;39(1):105-18. DOI: 10.5327/Z0100-0233-2015390100010

28. Mekuria AB, Gebresillassie BM, Erku DA, Haile KT, Birru EM. Knowledge and self-reported practice of insulin injection device disposal among diabetes patients in Gondar Town, Ethiopia: a cross-sectional study. J Diabetes Res. 2016;2016:1897517. DOI: http://dx.doi. org/10.1155/2016/1897517

29. Maia MA, Reis IA, Torres HC. Relationship between the users contact time in educational programs on diabetes mellitus and self-care skills and knowledge. Rev Esc Enferm USP. 2016;50(1):59-64. DOI: http://dx.doi.org/10.1590/S0080-623420160000100008

30. Sampaio HAC, Carioca AAF, Sabry MOD, Santos PM, Coelho MAM, Passamai MPB. Letramento em saúde de diabéticos tipo 2: fatores associados e controle glicêmico. Ciênc Saúde Coletiva. 2015;20(3):865-74. DOI: http://dx.doi.org/10.1590/1413-81232015203.12392014 